\title{
Formation of TRAPPIST-1 and other compact systems
}

\author{
Chris W. Ormel, Beibei Liu (刘倍贝), and Djoeke Schoonenberg
}

\begin{abstract}
Anton Pannekoek Institute (API), University of Amsterdam, Science Park 904,1090 GE Amsterdam, The Netherlands e-mail: [c.w.ormel;b.liu;d.schoonenberg]@uva.nl
\end{abstract}

Received 20 March 2017 / Accepted 19 May 2017

\begin{abstract}
TRAPPIST- 1 is a nearby $0.08 M_{\odot}$ M-star that was recently found to harbor a planetary system of at least seven Earth-sized planets, all within $0.1 \mathrm{au}$. The configuration confounds theorists as the planets are not easily explained by either in situ or migration models. In this paper we present a scenario for the formation and orbital architecture of the TRAPPIST-1 system. In our model, planet formation starts at the $\mathrm{H}_{2} \mathrm{O}$ iceline, where pebble-sized particles whose origin is the outer disk accumulate to trigger streaming instabilities. After their formation, planetary embryos quickly mature by pebble accretion. Planet growth stalls at Earth masses, where the planet's gravitational feedback on the disk keeps pebbles at bay. Planets are transported by type I migration to the inner disk, where they stall at the magnetospheric cavity and end up in mean motion resonances. During disk dispersal, the cavity radius expands and the innermost planets escape resonance. We argue that the model outlined here can also be applied to other compact systems and that the many close-in super-Earth systems are a scaled-up version of TRAPPIST-1. We also hypothesize that few close-in compact systems harbor giant planets at large distances, since they would have stopped the pebble flux from the outer disk.
\end{abstract}

Key words. planets and satellites: formation - planets and satellites: dynamical evolution and stability - methods: analytical planet-disk interactions

\section{Introduction}

TRAPPIST-1, a late-type $0.08 M_{\odot} \mathrm{M}$ star situated at a distance of $12 \mathrm{pc}$, is known to harbor an ultra-compact planetary system of at least six planets (Gillon et al. 2016, 2017). The seventh planet, TRAPPIST-1h, was recently confirmed by a transit timing analysis and $K 2$ data (Luger et al. 2017). All planets are within 0.1 au of their host star. Remarkably, all planets have masses similar to Earth, and the inferred densities, although highly uncertain, are consistent with rocky compositions (Gillon et al. 2017). The orbital period ratios of the TRAPPIST-1 planets indicate that planets $\mathrm{d} / \mathrm{e}, \mathrm{e} / \mathrm{f}$, and $\mathrm{g} / \mathrm{h}$ are very close to $3: 2$ mean motion resonance (MMR), while f/g shows a 4:3 commensurability. Planets b/c and $\mathrm{c} / \mathrm{d}$ are located somewhat farther from a first-order MMR.

The ultra-compact configuration of the TRAPPIST-1 system raises the question how it was formed. In situ formation, where rocky planets emerge from a giant impact phase (e.g., Hansen \& Murray 2012), would have required an unusually dense disk and would also not easily explain the resonant configuration. Planet migration seems to be a more plausible model (Lee \& Peale 2002). However, formation beyond the iceline cannot explain the predominantly rocky composition. In addition, traditional formation scenarios fail to explain why all planets end up at masses approximately equal to Earth's.

In this paper we explore a different scenario: it was the planetary building blocks in the form of millimeter- or centimetersized particles (pebbles) that migrated to the inner disk. Circumstellar disks contain large amounts of pebble-sized particles (e.g., Testi et al. 2014; Pérez et al. 2015), and the thermal emission from these particles has also been observed around lowmass stars or even brown dwarfs (Ricci et al. 2012). We argue that these pebbles were transformed into planetary embryos at the $\mathrm{H}_{2} \mathrm{O}$ iceline, situated at $\approx 0.1 \mathrm{au}$, where they migrated inward by type I migration. Interior to the $\mathrm{H}_{2} \mathrm{O}$ iceline, the planets grew by accretion of rocky pebbles. Inward planet migration ceased at the disk's inner edge, where they migrated into first-order MMR. Later, during the disk dispersion phase, the 3:2 MMRs of the inner two planet pairs were broken, resulting in the architecture that we witness today.

The goal of this paper is to present a synopsis of the early history of the TRAPPIST-1 system from simple analytical reasoning, which may inspire future numerical and more precise treatments. The adopted disk and stellar parameters (Table 1) have been optimized toward TRAPPIST-1 but are not out of the ordinary. In Sect. 2 we discuss the circumstellar disk of TRAPPIST-1. Section 3 presents the chronology. In Sect. 4 we speculate about the implications of our model for other stars.

\section{TRAPPIST-1 disk}

\subsection{Disk structure}

We assume that the TRAPPIST-1 circumstellar disk can be divided into two regions.

- The inner disk, $r \ll 1 \mathrm{au}$. This is the region where the iceline is located and where the planetary system forms. We furthermore assume that the inner disk is viscously relaxed, characterized by an $\alpha$-viscosity, $v=\alpha h^{2} r^{2} \Omega$, where $h$, the aspect ratio, is assumed constant and $\Omega_{\mathrm{K}}(r)$ is the local orbital frequency. The gas surface density $\Sigma_{\mathrm{g}}$ follows from the accretion rate $\dot{M}_{\mathrm{g}}: \Sigma_{\mathrm{g}}=\dot{M}_{\mathrm{g}} / 3 \pi v$ (Lynden-Bell \& Pringle 1974), where we adopt a value of $\dot{M}_{\mathrm{g}}=10^{-10} M_{\odot} \mathrm{yr}^{-1}$ typical for M stars (e.g., Manara et al. 2015). The constant aspect ratio of the inner disk is motivated by viscous heating and lamppost heating $\left(R_{\star} / r \sim 0.1\right.$; Rafikov \& De Colle 2006), as well as from spectral energy distribution (SED) fitting 
Table 1. Default disk and stellar parameters of TRAPPIST-1 during the planet formation phase.

\begin{tabular}{llll}
\hline \hline Symbol & Description & Value & Comments \\
\hline$\alpha$ & viscosity parameter inner disk & $10^{-3}$ & (a) \\
$\delta_{\text {ice }}$ & fractional width of iceline & 0.05 & \\
$\gamma_{\mathrm{I}}$ & prefactor in type-I migration rate & 4 & (b) \\
$\xi$ & $\# e$-foldings to reach pebble size & 10 & \\
$\tau_{\mathrm{p}}$ & pebble dimensionless stopping time exterior to iceline & 0.05 & (c) \\
$\zeta$ & dust fraction in pebbles & 0.5 & \\
$h$ & inner disk aspect ratio & 0.03 & \\
$r_{\text {out }}$ & outer disk radius & $200 \mathrm{au}$ & \\
$B_{\star}$ & stellar magnetic field strength at surface & $180 \mathrm{G}$ & \\
$M_{\star}$ & stellar mass & $0.08 M_{\odot}$ & \\
$M_{\text {disk }}$ & disk mass (gas) & $0.04 M_{\star}$ & \\
$M_{\mathrm{g}}$ & accretion rate & $10^{-10} M_{\odot} \mathrm{yr}^{-1}$ & \\
$R_{\star}$ & stellar radius & $0.5 R_{\odot}$ & \\
$Z_{0}$ & disk metallicity (global solids-to-gas mass ratio) & 0.02 & \\
\hline
\end{tabular}

Notes. ${ }^{(a)}$ Our default model. We also discuss more laminar and more turbulent disks $\left(10^{-4} \leq \alpha \leq 10^{-2}\right){ }^{(b)}$ Kley \& Nelson (2012); ${ }^{(c)}$ the stopping time of pebbles interior to the iceline is denoted $\tau_{\mathrm{s}}$ with $\tau_{\mathrm{s}} \ll \tau_{\mathrm{p}}$.

(Mulders \& Dominik 2012). The temperature structure corresponding to $h=0.03$ reads

$$
T(r)=180 \mathrm{~K} \frac{M_{\star}}{0.08 M_{\odot}}\left(\frac{h}{0.03}\right)^{2}\left(\frac{r}{0.1 \mathrm{au}}\right)^{-1} .
$$

- The outer disk, $r \gg 1$ au (Fig. 1). This region dominates the disk mass (in solids as well as gas). Since viscous timescales $\left(\sim r^{2} / v\right)$ are longer than the duration of the planet formation process (see Eq. (6)), a steady accretion disk is inappropriate. Instead, we simply adopt a power-law profile for the surface density:

$\Sigma_{\mathrm{g}, \text { out }}=\frac{(2-p) M_{\mathrm{disk}}}{2 \pi r_{\text {out }}^{2}}\left(\frac{r}{r_{\text {out }}}\right)^{-p} ; \quad(p<2)$,

where $M_{\text {disk }}$ is the total disk mass and $r_{\text {out }}$ the disk's outer radius. We choose $p=1, M_{\text {disk }}=0.04 M_{\star}$ and a metallicity of $Z_{0}=0.02$, which amounts to a total mass of $\approx 22$ Earth masses in solids, of which $\approx 11 M_{\oplus}$ is in rocky material (assuming a dust fraction in pebbles of $\zeta=0.5$; Lodders 2003).

The inner disk is truncated at the magnetospheric cavity radius (e.g., Frank et al. 1992):

$r_{\mathrm{c}}=\left(\frac{B_{\star}^{4} R_{\star}^{12}}{4 G M_{\star} \dot{M}_{\mathrm{g}}^{2}}\right)^{1 / 7} \approx 0.0102 \mathrm{au}$,

where $B_{\star}$ is the strength of the magnetic field measured at the surface of the star $\left(R_{\star}\right)$. In this and other equations, the numerical value follows from inserting the default parameters listed in Table 1 . Here, the surface magnetic field strength of $180 \mathrm{G}$, perhaps lower than the typical $\sim \mathrm{kG}$ of T Tauri stars, is consistent with observations of brown dwarfs (Reiners et al. 2009).

In general, disks feature a negative radial pressure gradient, which causes gas to rotate slower than Keplerian by an amount $\eta v_{\mathrm{K}}$ (Nakagawa et al. 1986). For our choices of $\Sigma_{\mathrm{g}}(r)$ and $T(r)$ for the inner disk, we obtain that $\eta=\frac{5}{4} h^{2}$ is constant. The subKeplerian motion induces the orbital decay of pebble-sized particles (Weidenschilling 1977): $v_{r}=-2 \eta v_{\mathrm{K}} \tau_{\mathrm{p}} /\left(1+\tau_{\mathrm{p}}^{2}\right) \approx-2 \eta v_{\mathrm{K}} \tau_{\mathrm{p}}$ where $\tau_{\mathrm{p}}=t_{\text {stop }} \Omega_{\mathrm{K}}$, the dimensionless stopping time, is assumed to be smaller than unity. We assume that just exterior

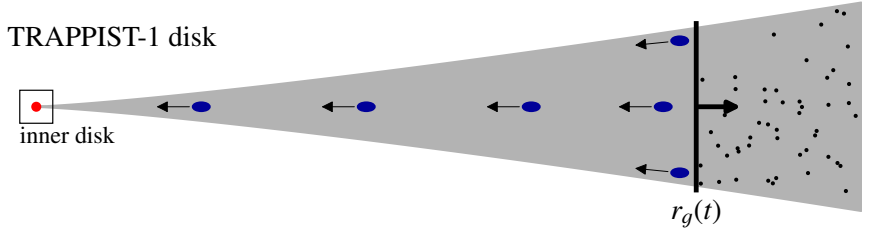

Fig. 1. TRAPPIST-1 outer disk. Dust grains coagulate and drift inward, resulting in a pebble front $r_{\mathrm{g}}$ that moves outward with time. After $t=$ $10^{5} \mathrm{yr}$, the pebble front reaches $50 \mathrm{au}$.

to the iceline $\tau_{\mathrm{p}}=0.05$, a value typical for drifting pebbles (Birnstiel et al. 2012; Lambrechts \& Johansen 2014; Krijt et al. 2016). For our standard disk parameters, $\tau_{\mathrm{p}}=0.05$ at 0.1 au corresponds to a physical size of $4 \mathrm{~cm}$. Interior to the iceline, the size (and $\tau_{\mathrm{p}}$ ) will be smaller because icy pebbles disintegrate.

\subsection{Pebble mass flux}

Most of the solids are located in the outer disk, initially in micron-sized grains. Using an $n-\sigma-\Delta v$ calculation, it follows that the collision timescales among grains is $t_{\text {coll }} \sim\left(Z_{0} \Omega_{\mathrm{K}}\right)^{-1}$, where $Z_{0}$ is the initial dust-to-gas ratio. Growth to pebble-sized particles, that is, large enough to start drifting, involves many binary collisions. Let $t_{\mathrm{peb}}=\xi\left(Z_{0} \Omega_{\mathrm{K}}\right)^{-1}$, with $\xi \approx 10$ (Krijt et al. 2016), be the time needed before drifting pebbles appear. By equating $t=t_{\mathrm{peb}}$ and solving for $r$, we find the radius of the pebble front $r_{\mathrm{g}}$, where the particles reach their drifting size:

$r_{\mathrm{g}}=\left(\frac{G M_{\star} Z_{0}^{2} t^{2}}{\xi^{2}}\right)^{1 / 3}=50 \mathrm{au}\left(\frac{t}{10^{5} \mathrm{yr}}\right)^{2 / 3}$.

The advancing $r_{\mathrm{g}}(t)$ generates pebbles at a rate $\dot{M}_{P}=$ $2 \pi r_{\mathrm{g}} \dot{r}_{\mathrm{g}} Z_{0} \Sigma_{\mathrm{g}}\left(r_{\mathrm{g}}\right)$ and results in a pebble-to-gas mass flux ratio of

$\mathcal{F}_{\mathrm{p} / \mathrm{g}} \equiv \frac{\dot{M}_{\mathrm{p}}}{\dot{M}_{\mathrm{g}}}=\frac{2 M_{\mathrm{disk}} Z_{0}^{5 / 3}}{3 \dot{M}_{\mathrm{g}} r_{\mathrm{out}} \xi^{2 / 3}}\left(\frac{G M_{\star}}{t}\right)^{1 / 3} \approx 1.1\left(\frac{t}{10^{5} \mathrm{yr}}\right)^{-1 / 3}$

(cf. Lambrechts \& Johansen 2014). The pebble flux will disappear after the time $t_{\mathrm{end}}$, when the pebble front hits the outer edge 


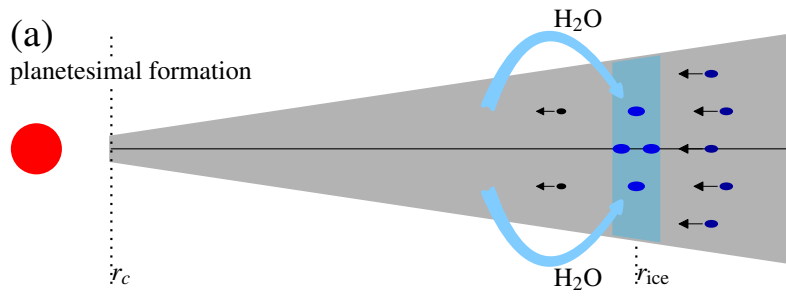

(b)

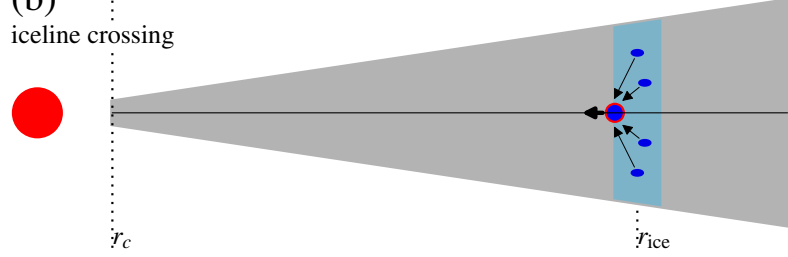

(c)

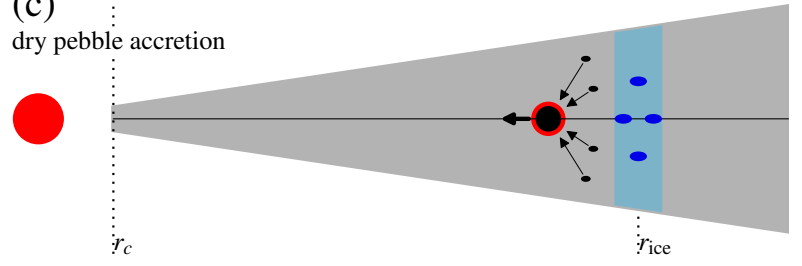

(d)

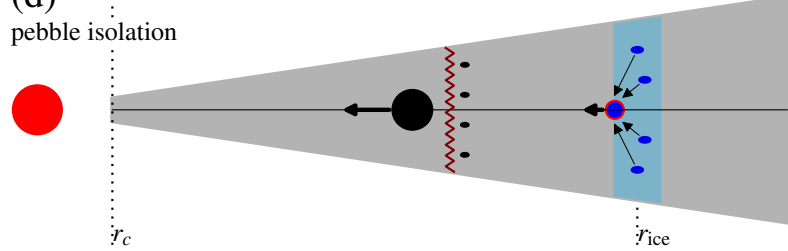

(e) $\quad: 3: 3: 3: 3 \stackrel{3: 2}{\longrightarrow} \stackrel{3: 2}{\longleftrightarrow} \stackrel{4: 3}{\longleftrightarrow} \longleftarrow 3: 2$

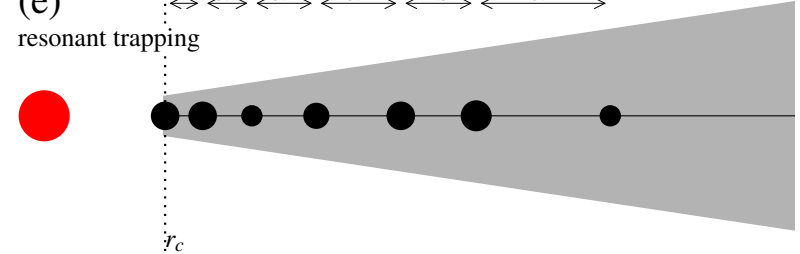

(f)

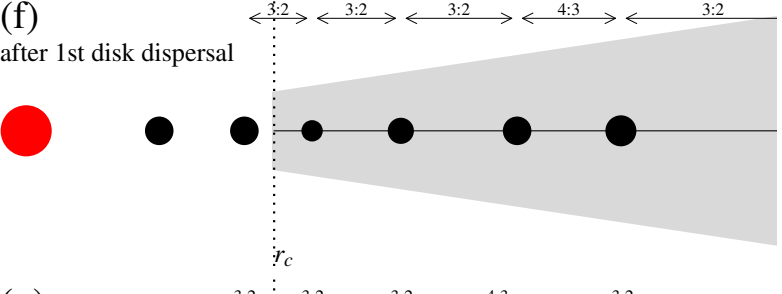

(g)

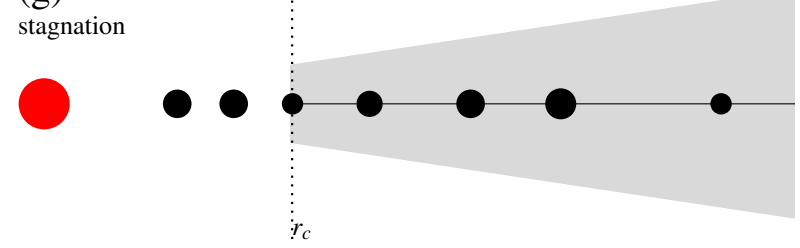

(h)

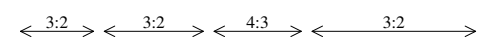

after final dispersal

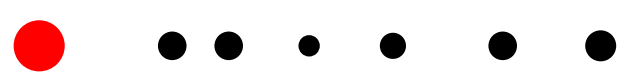

Fig. 2. Stages in the formation of the TRAPPIST-1 system. Panels show the inner disk ( $r \lesssim 0.1 \mathrm{au})$ : the left panels describe the formation phases, and the right panels the disk dispersion and dynamical rearrangement. Stages are described in Sect. 3. Sizes and aspect ratios are not to scale, but relative distances are.

of the disk, $r_{\mathrm{g}}=r_{\text {out }}$ :

$t_{\text {end }}=\frac{\xi}{Z_{0}} \sqrt{\frac{r_{\text {out }}^{3}}{G M_{\star}}} \approx 8 \times 10^{5} \mathrm{yr}$.

\section{Synopsis}

In our scenario, the formation of the TRAPPIST-1 planets proceeds in two stages. In the first stage, planetary embryos assemble sequentially at the iceline, migrate inward, and end up in resonance near the disk edge (Fig. 2, panels a-d). The second stage concerns the dynamical rearrangement, triggered by the disk dispersal, which moves the inner planets out of MMR (panels e-h).

In the first stage, our assumption is that planets form sequentially, not simultaneously. In our model we assume that the $\mathrm{H}_{2} \mathrm{O}$ iceline is the location where the midplane solids-to-gas ratio exceeds unity, triggering streaming instabilities and spawning the formation of planetesimals. These planetesimals merge into a planetary embryo, whose growth is aided by icy pebble accretion. Once its mass becomes sufficiently large, it migrates interior to the $\mathrm{H}_{2} \mathrm{O}$ iceline by type I migration, where it continues to accrete (now dry) pebbles until it reaches the pebble isolation mass. After some time, a second embryo forms at the snowline, which follows a similar evolutionary path as its predecessor. Even though the inner planet's growth could be reduced by its younger siblings' appetite for pebbles, it always remains ahead in terms of mass. Planet migration stalls at the inner disk edge, where the planets are trapped in resonance.

\subsection{Formation of planetesimals (a)}

The first step is the concentration of pebble-sized particles and their subsequent gravitational collapse into planetesimals. A prominent mechanism is the streaming instability, where particles clump into filaments because of the backreaction of the solids on the gas (Youdin \& Goodman 2005). These filaments fragment and spawn planetesimals (Johansen et al. 2007). Recent work has demonstrated that streaming instabilities can be triggered for a broad range of stopping times (Yang et al. 2017); however, a prerequisite is that the solids-to-gas ratio must be substantial (Johansen et al. 2009; Carrera et al. 2015; Yang et al. 2017). Since streaming instabilities arise by virtue of the backreaction of the solids on the gas, we seek volume solids-to-gas ratios $\rho_{\mathrm{p}} / \rho_{\mathrm{g}} \sim 1$.

However, the inside-out growth and drift of solids do not guarantee high solids-to-gas ratios (Birnstiel et al. 2010; Krijt et al. 2016; Sato et al. 2016). The midplane pebble-to-gas density ratio is only

$\left(\frac{\rho_{\mathrm{p}}}{\rho_{\mathrm{g}}}\right)_{\text {midplane }}=\frac{\Sigma_{\mathrm{p}} / H_{\mathrm{p}}}{\Sigma_{\mathrm{g}} / H_{\mathrm{g}}}=\frac{3 \mathcal{F}_{\mathrm{p} / \mathrm{g}}}{5} \sqrt{\frac{\alpha}{\tau_{\mathrm{p}}}} \approx 0.08 \mathcal{F}_{\mathrm{p} / \mathrm{g}}$,

where we used the $\alpha$-disk model for $\Sigma_{g}$ (Sect. 2.1), $\Sigma_{\mathrm{p}}=$ $\mathcal{F}_{\mathrm{p} / \mathrm{g}} \dot{M}_{\mathrm{g}} / 2 \pi r v_{r}$ with $v_{r} \simeq 2 \tau_{\mathrm{p}} \eta v_{\mathrm{K}}$, and a pebble-to-gas scaleheight of $H_{\mathrm{p}} / H_{\mathrm{g}}=\sqrt{\tau_{\mathrm{p}} / \alpha}$ (Dubrulle et al. 1995). Therefore, the particle mass-loading is unlikely to approach unity.

To further enhance $\rho_{\mathrm{p}} / \rho_{\mathrm{g}}$, we invoke the $\mathrm{H}_{2} \mathrm{O}$ iceline, located at $\approx 0.1$ au. Recently, we have demonstrated that enhancements 


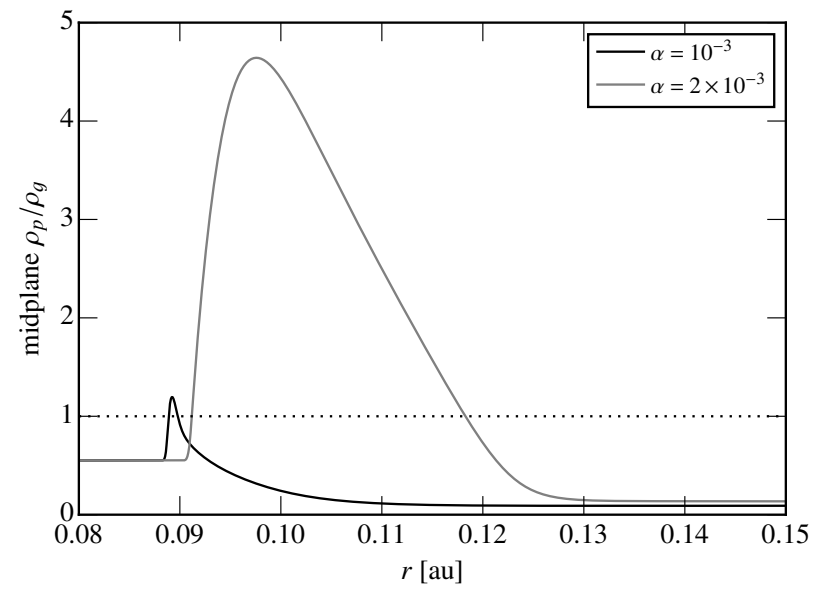

Fig. 3. Midplane solids-to-gas ratio for the many-seeds model design of Schoonenberg \& Ormel (2017) with $\mathcal{F}_{\mathrm{p} / \mathrm{g}}=1.1$ for $\alpha=10^{-3}$ and $2 \times 10^{-3}$. The strong boost in solids-to-gas between the two runs arises from the backreaction of the solids on the gas, which reduces the radial drift of the particles, resulting in a more significant pileup.

of up to 10 can be attained (Schoonenberg \& Ormel 2017) ${ }^{1}$. Figure 3 presents the result of the Schoonenberg \& Ormel (2017) "many-seeds" model for the iceline of TRAPPIST-1 disk. Owing to diffusion, $\mathrm{H}_{2} \mathrm{O}$ vapor is transported across the iceline, where it condenses on the incoming pebbles, creating a distinct bump in the midplane pebble-to-gas ratio. For our standard parameters $\left(\alpha=10^{-3}\right)$ we find that $\rho_{\mathrm{p}} / \rho_{\mathrm{g}}$ just exceeds unity, triggering the formation of planetesimals. The pebble-to-gas ratio increases as function of $\alpha$ for several reasons: (i) more $\mathrm{H}_{2} \mathrm{O}$ vapor is being transported at larger $\alpha$; (ii) the base $\rho_{\mathrm{p}} / \rho_{\mathrm{g}}$ (Eq. (7)) increases with $\alpha$; (iii) the backreaction of the solids on the gas, which starts to become important at $\rho_{\mathrm{p}} / \rho_{\mathrm{g}} \sim 1$, reduces the particle drift, which strongly enhances the pileup effect. Finally, in Fig. 3 increasing $\alpha$ by a factor two incidentally coincides with the Epstein-Stokes drag transition, which causes another jump (Schoonenberg \& Ormel 2017).

On the other hand, for $\alpha<10^{-3}$ pebble-to-gas ratios are likely to stay much below unity. However, disintegration of icy pebbles releases micron-sized silicate grains, which can create strong pileups just interior to $r_{\text {ice }}$ (Saito \& Sirono 2011). Ida \& Guillot (2016) found that the midplane mass-loading of (silicate) grains is high enough to trigger direct gravitational instability, especially at low $\alpha$. Even when particles recoagulate, however, their size is likely to be limited by collisional fragmentation or bouncing (e.g., Güttler et al. 2010), resulting in shorter stopping times and dust-to-gas ratios high enough to trigger streaming instability (Banzatti et al. 2015; Drążkowska et al. 2016). In these cases planets start out rocky, interior to the iceline, and the phase described in the next subsection does not exist.

\subsection{Migration interior to iceline (b)}

We assume that a single dominant planetary embryo emerges from the planetesimal-pebble mix. Its growth is indeed very

\footnotetext{
1 Stevenson \& Lunine (1988), and more recently, Ros \& Johansen (2013), have pointed to the $\mathrm{H}_{2} \mathrm{O}$ iceline to trigger planetesimal formation and their subsequent growth. However, their models considered closed systems where either the particles or the gas was not removed from the system. In contrast, the steady-state model of Schoonenberg \& Ormel (2017) features a constant inward mass flux of ice and gas.
}

rapid:

$t_{\text {grow }}=\frac{M_{\mathrm{pl}}}{\epsilon_{\mathrm{PA}} \mathcal{F}_{\mathrm{p} / \mathrm{g}} \dot{M}_{\mathrm{g}}}=\frac{q_{\mathrm{pl}} M_{\star}}{\epsilon_{\mathrm{PA}} \mathcal{F}_{\mathrm{p} / \mathrm{g}} \dot{M}_{\mathrm{g}}}=\frac{8 \times 10^{3} \mathrm{yr}}{\mathcal{F}_{\mathrm{p} / \mathrm{g}} \epsilon_{\mathrm{PA}}}\left(\frac{q_{\mathrm{pl}}}{10^{-5}}\right)$,

where $q_{\mathrm{pl}}=M_{\mathrm{pl}} / M_{\star}$ and $\epsilon_{\mathrm{PA}}$ is the pebble accretion efficiency. We evaluate $\epsilon_{\mathrm{PA}}$ in Sect. 3.3.

Planets migrate inward at a rate $r / t_{\mathrm{I}}$, where $t_{\mathrm{I}}$ is the type I migration time:

$t_{\mathrm{I}}=\frac{h^{2}}{\gamma_{\mathrm{I}} q_{\mathrm{p}} q_{\mathrm{gas}} \Omega_{\mathrm{K}}}=\frac{3 \pi \alpha h^{4}}{\gamma_{\mathrm{I}} q_{\mathrm{pl}} \dot{M}_{\mathrm{g}} / M_{\star}}=1.5 \times 10^{5}\left(\frac{q_{\mathrm{pl}}}{10^{-5}}\right)^{-1} \mathrm{yr}$,

where $q_{\text {gas }}=\Sigma_{\mathrm{g}} r^{2} / M_{\star}$ and $\gamma_{\mathrm{I}}$ is on the order of unity (Tanaka et al. 2002; Kley \& Nelson 2012). The planet crosses the iceline when $t_{\text {grow }} \approx \delta_{\text {ice }} t_{\mathrm{I}}$, where $\delta_{\text {ice }}=\delta r_{\text {ice }} / r$ is the fractional width of the iceline. We therefore find that the embryo moves interior to the iceline at a mass

$M_{\text {cross }}=\sqrt{\frac{3 \pi \alpha \delta_{\text {ice }} \mathcal{F}_{\mathrm{p} / \mathrm{g}} \epsilon_{\mathrm{PA}}}{\gamma_{\mathrm{I}}}} h^{2} M_{\star}=0.26 M_{\oplus} \sqrt{\mathcal{F}_{\mathrm{p} / \mathrm{g}} \epsilon_{\mathrm{PA}}}$.

With $\mathcal{F}_{\mathrm{p} / \mathrm{g}}=1.1$ and $\epsilon_{\mathrm{PA}}=0.1$ (motivated in Sect. 3.3), this corresponds to a Mars-mass embryo of which $\approx 50 \%$ is of icy composition. The remainder of the accretion takes place in the interior region, however, where pebbles are dry, and the planet ends up predominantly rocky.

We note that the value of $M_{\text {cross }}$ depends considerably on the viscosity parameter $\alpha$. A larger $\alpha$ implies a lower gas density, suppressing migration, and a thicker ice "line" ( $\delta_{\text {ice }}$ is larger; Schoonenberg \& Ormel 2017), promoting a prolonged stay in the ice-rich region. For $\alpha \gtrsim 10^{-2}$ the crossover mass can be similar to the pebble isolation mass (see Sect. 3.4), implying that the planet has a high $\mathrm{H}_{2} \mathrm{O}$ content.

\subsection{Efficient pebble accretion (b, $c)$}

Planetesimals formed by streaming instability can have sizes of up to $\sim 100 \mathrm{~km}$ (Simon et al. 2016; Schäfer et al. 2017). These planetesimals will accrete the pebbles that are drifting from the outer disk, in so-called settling interactions (Ormel \& Klahr 2010). This mechanism, more popularly known as pebble accretion (Lambrechts \& Johansen 2012), is particularly attractive in case of TRAPPIST-1 because it is highly efficient: a large fraction of the pebbles are accreted.

First, consider pebble accretion exterior to the iceline. We assume that pebble accretion operates in the planar (2D) mode, which is appropriate for low-to-modest $\alpha$. Accretion of pebbles in the planar mode amounts to a rate of (Ida et al. 2016; Ormel 2017)

$\dot{M}_{\mathrm{pl}} \sim 2 R_{\mathrm{Hill}}^{2} \Omega_{\mathrm{K}} \tau_{\mathrm{p}}^{2 / 3} \Sigma_{\mathrm{p}}$,

where $R_{\text {Hill }}=r\left(q_{\mathrm{pl}} / 3\right)^{1 / 3}$ is the Hill radius. Since the pebble flux is $(2 \pi r) v_{r} \Sigma_{\mathrm{p}}$, pebbles are accreted at an efficiency of

$\epsilon_{\mathrm{PA}} \sim \frac{2}{5 \times 3^{2 / 3} \pi \tau_{\mathrm{p}}^{1 / 3}}\left(\frac{q_{\mathrm{pl}}}{h^{3}}\right)^{2 / 3}=0.1\left(\frac{q_{\mathrm{pl}}}{10^{-5}}\right)^{2 / 3}$,

where we inserted $v_{r} \approx \frac{5}{2} h^{2} \tau_{\mathrm{p}} \Omega_{\mathrm{K}} r$ (Sect. 2). In a more precise $N$ body calculation (Liu \& Ormel, in prep.), we find $\epsilon_{\mathrm{PA}}=0.25$ for $q_{\mathrm{pl}}=10^{-5}$. Compared to solar-type stars, pebble accretion for the TRAPPIST-1 disk is particularly efficient because the disk is thin (pebbles are accreted in the 2D limit) and Hill radii are larger as a result of the low stellar mass. 
Next, consider pebble accretion interior to the iceline. Although the grains liberated by sublimating icy pebbles are likely to have recoagulated, their sizes are much smaller because of the silicate fragmentation threshold. Therefore, $\tau_{\mathrm{s}} \ll \tau_{\mathrm{p}}$ ("s" referring to silicate pebbles) and pebble accretion operates in the $3 \mathrm{D}$ limit (Ida et al. 2016; Ormel 2017),

$\dot{M}_{\mathrm{pl}-3 \mathrm{D}} \sim 6 \pi R_{\mathrm{Hill}}^{3} \tau_{\mathrm{s}} \Omega \rho_{\mathrm{s}}$,

where $\rho_{\mathrm{s}}$ is the midplane density of silicate pebbles. In the limit where silicate pebbles are distributed over the entire gas scaleheight $\rho_{\mathrm{s}}=\Sigma_{\mathrm{s}} / 2 h r$, with $\Sigma_{\mathrm{s}}$ the silicate surface density interior to the iceline, we obtain an efficiency of

$\epsilon_{\mathrm{PA}-3 \mathrm{D}}=\frac{\dot{M}_{\mathrm{pl}-3 \mathrm{D}}}{(2 \pi r)\left(2 \eta \tau_{\mathrm{s}} r \Omega\right) \Sigma_{\mathrm{s}}} \sim \frac{1}{5} \frac{q_{\mathrm{pl}}}{h^{3}}=0.07\left(\frac{q_{\mathrm{pl}}}{10^{-5}}\right)$.

This estimate is conservative: settling increases efficiencies by a factor $\sqrt{\tau_{\mathrm{s}} / \alpha}$ and numerically obtained rates are typically higher by a factor two ${ }^{2}$. Hence, growth remains rapid; from Eq. (8) we obtain a growth time of $t_{\text {grow }} \sim 10^{5} \mathrm{yr} / \mathcal{F}_{\mathrm{s} / \mathrm{g}}$, where $\mathcal{F}_{\mathrm{s} / \mathrm{g}}$ is now the silicate-to-gas mass flux ratio.

Our results differ in two aspects from those of Morbidelli et al. (2015), who also calculated pebble accretion interior and exterior to the iceline. Morbidelli et al. (2015) kept embryos at fixed locations (no migration), and the outer embryo, which grew faster, was seen to ultimately starve the inner disk from pebbles. In our scenario there is no true competition because of the aforementioned sequential growth and migration. The second difference is that $q_{\mathrm{pl}} / h^{3}$, which enters the efficiency expressions at different powers (Eq. (12) vs. Eq. (14)), is much larger in TRAPPIST-1. When embryos cross the iceline, we have $q_{\mathrm{pl}} / h^{3} \sim 0.1$, whereas Morbidelli et al. (2015) started from $q_{\mathrm{pl}} / h^{3} \sim 10^{-4}$. Therefore, the $2 \mathrm{D}$ rate (exterior to the iceline) in Morbidelli et al. (2015) is much higher than the 3D rates (interior), whereas in the case of TRAPPIST-1 the pebble accretion efficiencies are similar.

\subsection{Pebble isolation (d)}

Pebble accretion terminates when the gravitational feedback of the planet on the disk becomes important. At regions where pressure maxima emerge, pebbles stop drifting. Essentially, pebble isolation describes the onset of gap opening. While the pebble isolation mass for disks around solar-type stars at 5 au is $\approx 20 M_{\oplus}$ (Lambrechts et al. 2014), it will be much lower at the iceline of the TRAPPIST-1 disk. A necessary condition for gap opening is that the Hill radius exceeds the disk scaleheight, $q_{\mathrm{pl}}=M_{\mathrm{pl}} / M_{\star}>h^{3}$ (Lin \& Papaloizou 1993), therefore

$M_{\mathrm{p}, \text { iso }} \sim h^{3} M_{\star}=0.72 M_{\oplus}$.

These arguments have motivated us to adopt $h \approx 0.03$, but this choice is not unreasonable. The fact that Earth-mass planets naturally emerge from the pebble-driven growth scenario is a distinctive feature of the model.

After the innermost planet first reaches the pebble isolation mass, silicate pebbles no longer accrete on TRAPPIST-1. From

\footnotetext{
2 On the other hand, when $\tau_{\mathrm{s}}<\alpha$, Eq. (14) will be an overestimate because the particle radial motion is then determined by the radial velocity of the gas. Since the inner disk is viscously relaxed, however, high $\alpha$ in turn implies larger $\tau_{\mathrm{s}}$, so the situation where $\tau_{\mathrm{s}} \ll \alpha$ is not easily retrieved. For example, for a silicate pebble size of $1 \mathrm{~mm}$ and an internal density of $3 \mathrm{~g} \mathrm{~cm}^{-3}$, we obtain $\tau_{\mathrm{s}} / \alpha \approx 1.1$ for our standard parameters at $r=0.05$ au.
}

that point on, the entire silicates mass reservoir, except for the grains that are so tiny that they follow the gas (Zhu et al. 2012), is available for making planets, resulting in a high global formation efficiency. Before isolation has been reached, the innermost planet loses $\left(1-\epsilon_{\mathrm{PA}-3 \mathrm{D}}\right) / \epsilon_{\mathrm{PA}-3 \mathrm{D}}$ pebbles for every silicate pebble it accretes. Evaluating this number at the final mass of the planet ( 1 Earth mass or $q_{\mathrm{pl}}=3.7 \times 10^{-5}$ ), we obtain that $\sim 3$ Earth masses in silicates are lost. Our mechanism therefore efficiently turns solids into planets. An efficient mechanism is indeed necessary because the initial disk contains only 11 Earth masses in rocky materials (Sect. 2).

\subsection{Migration and resonance trapping $(d)-(e)$}

On timescales $\sim t_{\mathrm{I}}$, planets type I migrate to the disk edge $\left(r_{\mathrm{c}}\right)$. We assume here, for simplicity, that the migration is always inward, that is, no special thermodynamical effects are at work that could reverse the migration sign (Paardekooper et al. 2011; Benítez-Llambay et al. 2015). The processes illustrated in Figs. $2 a-c$ then repeat until a convoy of seven planets is established.

Convergent migration of planets naturally results in resonant trapping (Terquem \& Papaloizou 2007). For our disk model, planets are likely to be trapped in the $2: 1 \mathrm{MMR}$. According to Ogihara \& Kobayashi (2013), the condition to avoid trapping in the $2: 1$ resonance reads $^{3}$

$t_{\mathrm{I}}<t_{\mathrm{a}, \mathrm{crit}} \approx 4 \times 10^{3} \mathrm{yr}\left(\frac{r}{0.1 \mathrm{au}}\right)^{3 / 2}$.

However, most of the TRAPPIST-1 planets are observed near the 3:2 MMR. This implies that $t_{I}$ (Eq. (9)) is too long by a factor 10. A quantitative at 0.1 au and the disparity only increases with lower $r$. A quantitative model to explain the settling into the 3:2 MMR resonance is beyond the scope of this paper, but we offer several ideas for further investigation.

- The TRAPPIST-1 planets will form a resonant convoy, where all outer planets "push" on the innermost ones, effectively increasing $\gamma_{\mathrm{I}}$ and decreasing the migration timescale (McNeil et al. 2005). For a low- $\alpha$ disk $\left(\alpha=10^{-4}\right)$, the gas densities are also higher by a factor 10 , and we can expect planets to be moved across the 2:1.

- Stochastic forces, for example, triggered by density fluctuations in a magneto-rotational unstable (MRI) disk (Okuzumi \& Ormel 2013), can move planets across resonances (Paardekooper et al. 2013). Similarly, the disk accretion rate $\dot{M}_{\mathrm{g}}$, assumed constant here, is likely to vary in time (Hartmann 2009). At intervals where $\dot{M}$ peaks, the condition $t_{\mathrm{I}}<t_{\mathrm{a} \text {,crit }}$ can be met.

- At the time when the outer planet crosses the iceline, its period ratios with the inner planet happens to lie within the 2:1 and 3:2 resonance locations. Since $t_{\text {grow }} \leq t_{\mathrm{I}}$, it is indeed plausible that the inner planet will not have migrated very far. Nevertheless, a certain level of fine-tuning is required for the planets to start within the 3:2 and 2:1 window.

Investigating each of these scenarios requires a dedicated numerical simulations. In the following, we assume that the planets end up in MMR resonance, as shown in Fig. 2e. 


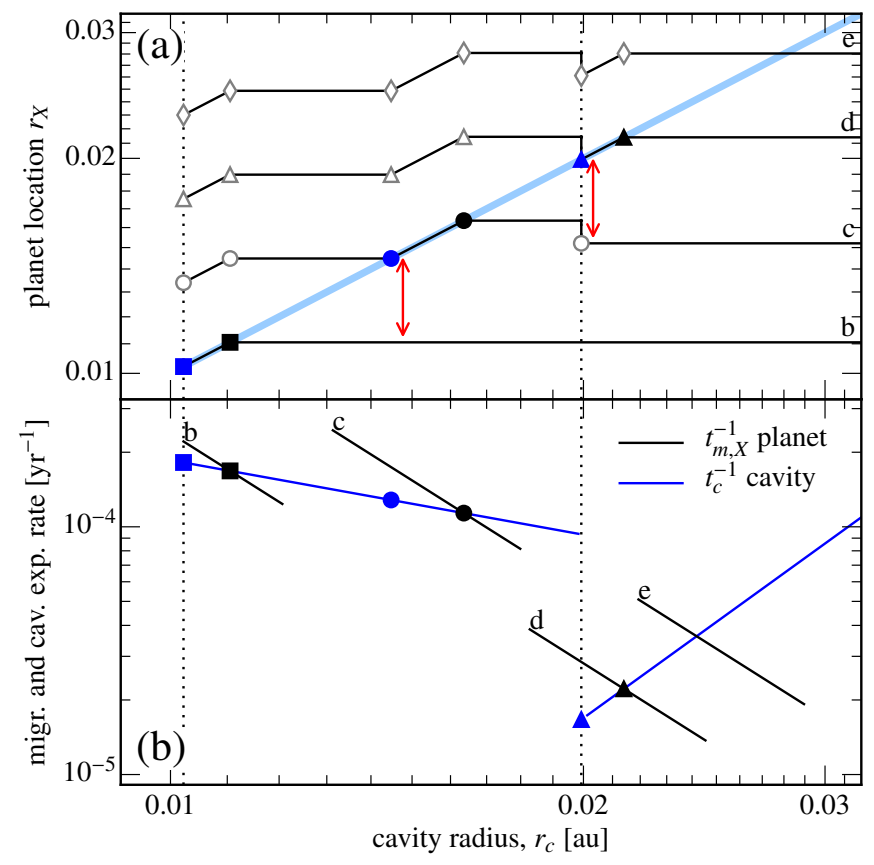

Fig. 4. Resonance escape. Bottom: one-sided migration rates $t_{m, \mathrm{X}}^{-1}$ as function of cavity radius $r_{\mathrm{c}}$ for planet $\mathrm{X}$ (black lines; labeled) and expansion rate of the magnetospheric cavity $t_{\mathrm{c}}^{-1}$ (blue lines). The form $t_{\mathrm{c}}\left(r_{\mathrm{c}}\right)$ is chosen to fit the orbital configuration of the TRAPPIST-1 system. The jump in $t_{\mathrm{c}}$ at $r_{\mathrm{c}}=0.02$ au indicates that $\dot{M}_{\mathrm{g}}$ and $r_{\mathrm{c}}(t)$ are temporarily constant. Blue symbols denote a planet that couples to (moves synchronously with) the cavity radius. Black symbols denote the point of decoupling (the planet falls into the cavity). Top: the location of the planets corresponding to $r_{\mathrm{c}}(t)$. Red arrows indicate the point where the $3: 2$ resonance is broken because of divergent migration.

\subsection{Disk dispersal and rebound (f)-(h)}

Planet pairs b/c and c/d are currently not near the 3:2 MMR. Several mechanisms have been proposed to move planets out of resonance, such as damping by stellar tides (Lithwick \& Wu 2012) or giant impacts (Ogihara et al. 2015). Here we consider the magnetospheric rebound mechanism (Liu et al. 2017), which relies on the outward movement of the stellar magnetospheric cavity $r_{\mathrm{c}}$ during disk dispersal. In principle, the innermost planet at $r=r_{\mathrm{c}}$ is tightly coupled to the disk by strong one-sided torques (Liu et al. 2017). It therefore tends to follow the expanding $r_{\mathrm{c}}$. When the cavity expansion is too rapid, however, that is, when the expansion rate is higher than the planet migration rate, the planet decouples and falls in the magnetospheric cavity.

We have tuned the detailed behavior of $r_{\mathrm{c}}(t)$ (or, equivalently, $\dot{M}(t)$ by Eq. (3)) in order to retrieve the orbital parameters of TRAPPIST-1. In Fig. 4a we plot the positions of planets b-e as a function of the (expanding) cavity radius $r_{\mathrm{c}}$ (for clarity we omit planets $\mathrm{f}, \mathrm{g}$, and $\mathrm{h}$ ). We note that $r_{\mathrm{c}}$ is used as a proxy for time. In Fig. $4 \mathrm{~b}$ we plot the rate at which the cavity expands $\left(t_{\mathrm{c}}^{-1}=\dot{r}_{\mathrm{c}} / r_{\mathrm{c}}\right.$, blue curve) and the maximum one-sided planet migration rates, $t_{m, \mathrm{X}}^{-1}$, for the planets $(\mathrm{b}, \mathrm{c}, \mathrm{d}$, or e):

$t_{\mathrm{m}, X}^{-1}=2 C_{\mathrm{hs}} q_{\mathrm{gas}}\left(\frac{q_{\mathrm{pl}}}{h^{3}}\right)^{1 / 2} \Omega_{\mathrm{K}} \propto C_{\mathrm{X}}\left(\frac{r_{\mathrm{c}}}{r_{\mathrm{c} 0}}\right)^{-3.5}$

(Liu et al. 2017). The key point is that for any planet $t_{\mathrm{m}, \mathrm{X}}^{-1} \propto \dot{M}_{\mathrm{g}} \propto$ $r_{\mathrm{c}}^{-3.5}$ (see Eq. (3)) and that it depends, through $C_{\mathrm{X}}$, on the planet

\footnotetext{
3 Here, we evaluated Eq. (4) of Ogihara \& Kobayashi (2013) for $M_{\mathrm{pl}}=$
} $M_{\oplus}$ and $M_{\star}=0.08 M_{\odot}$. properties: more massive planets tend to have larger $C_{\mathrm{X}}$, and $C_{\mathrm{X}}$ also decreases with the number of planets in a resonance chain. Here we take $t_{\mathrm{c}} \sim 10^{4} \mathrm{yr}$ (with a dependence on $r_{\mathrm{c}}$ as in Fig. 2b) and $C_{\mathrm{X}}=\{1.5,4,2,5\}$.

Our model features a two-stage disk dispersal. First, $r_{\mathrm{c}}$ doubles from 0.01 to $0.02 \mathrm{au}$, where it temporarily stalls (Fig. 2f). Planet $\mathrm{b}$ decouples relatively quickly from the expanding cavity front, whereas the more massive planet c couples longer to $r_{\mathrm{c}}$ as a result of its larger $C_{\mathrm{X}}$. The 3:2 resonance between planets b and $\mathrm{c}$ is broken because of divergent migration just after the coupling of $\mathrm{c}$ to the cavity radius.

During the time when $r_{\mathrm{c}}$ pauses at $0.02 \mathrm{au}$, the accretion rate $\dot{M}_{\mathrm{g}}$ is constant. We assume that this phase takes long enough for planet d to migrate inward, such that it now coincides with the cavity radius (Fig. 2g). Planets c, e, and f-h (not shown in Fig. 2) follow suit as they are still part of the resonance chain.

During the second dispersal phase, planet d only briefly couples to the expanding $r_{\mathrm{c}}$, but long enough to escape resonance with c. Afterward, the expansion proceeds too rapidly for planets to couple. For example, at the point where $r_{\mathrm{c}}$ meets planet $\mathrm{e}$ $\left(r_{\mathrm{c}} \approx 0.028 \mathrm{au}\right)$, the one-sided migration rate is at least a factor three lower than $\dot{r}_{\mathrm{c}}$. Therefore, e and the other outer planets stay in resonance.

In the above discussion, we have referred to "resonance" as exact commensurability (nominal resonance). However, planets moving away from exact commensurability can maintain librating resonant angles; Batygin \& Morbidelli (2013) found that dissipative divergence in this way keeps planets in resonance in the dynamical sense. Luger et al. (2017) have shown that the TRAPPIST-1 planets form a complex chain of three-body resonances ${ }^{4}$. Long-term dynamical stability would be greatly promoted when the initial libration width of the resonant angles is small (Tamayo et al. 2017) or when stellar tides play a role (Papaloizou 2015).

\section{Closing remarks}

We have outlined a new framework to understand the formation of planetary systems around very low-mass stars. With some modest tuning, we have succeeded in obtaining a system whose architecture reflects that of TRAPPIST-1 (Fig. 2h). Its most radical idea is that planets assemble at a specific location, that is, at the $\mathrm{H}_{2} \mathrm{O}$ iceline. This is in contrast to classical models, where planets form locally, as well as to population synthesis models, which do account for migration, but do not (yet) provide a physical model for the initial position of planetary embryos. Our scenario is more complete, as we provide a physical model where planetary embryos (first) form.

In design, our model much resembles the inside-out formation model by Chatterjee \& Tan (2014), which also acknowledges the role of drifting pebbles in spawning planets at a specific location. In the models of Chatterjee \& Tan (2014) and Hu et al. (2016), this transition occurs at the interface of an MRIactive and MRI-“dead" region, characterized by $T \sim 1200 \mathrm{~K}$, resulting in a pressure bump where pebbles stop drifting and planet formation proceeds by direct gravitational instability (Goldreich \& Ward 1973). Chatterjee \& Tan (2014) argued that planets, once formed, experience little migration and a second planet forms in close proximity, forming a tightly packed system consistent with the Kepler census.

\footnotetext{
4 From timing analysis it is much easier to identify a three-body resonance, for which only the mean longitude are important, rather than two-body resonance, which require the arguments of periapses.
} 
For TRAPPIST-1, however, the active:dead transition radius will lie very close to the disk inner edge, since temperatures and disk accretions rates for these tiny stars are much lower. A variant of their as well as our model would be to assume that pebbles drift all the way to the disk inner edge $r_{\mathrm{c}}$. Planets then grow up to their isolation mass $\left(\sim M_{\oplus}\right)$ near $r_{\mathrm{c}}$. However, in this case, planets will be much more tightly packed because pebbles do not stall at MMR but at the pressure bump, of which the typical distance is the gas disk scaleheight. It would be hard to produce the TRAPPIST-1 architecture from such compact conditions.

Several aspects of our narrative demand further investigation. We have mentioned the difficulty of avoiding the planets from becoming trapped in the 2:1 MMR because of the rather low disk mass (Sect. 3.5). Another key assumption of our scenario is that one embryo at a time emerges from the iceline. Simultaneous formation of multiple embryos could result in an excited system of small embryos, suppressing growth (Kretke \& Levison 2014; but see Levison et al. 2015). Because of the short dynamical timescales, it seems viable that bodies quickly coalesce, but this complex issue of how the streaming instability operates in the presence of planetary embryos warrants further investigation.

A prominent feature of our model is that planets mature in gas-rich disks; there is no need for a post-disk giant impact phase. Therefore, the TRAPPIST- 1 planets could have accreted primordial $\mathrm{H} / \mathrm{He}$ atmospheres, for which there currently is no indication (de Wit et al. 2016). However, planets close to their host star receive high doses of UV and X-ray fluxes (Stelzer et al. 2013; Bourrier et al. 2017), which efficiently strips their atmospheres away (Lopez et al. 2012). Composition-wise, the inferred densities of $3-6 \mathrm{~g} \mathrm{~cm}^{-3}$ (Gillon et al. 2017) rule out that these planets are entirely composed of $\mathrm{H}_{2} \mathrm{O}$. Given the error bars on the mass, however, planets are consistent with large $(\sim 10 \%) \mathrm{H}_{2} \mathrm{O}$ fractions, in particular planet $\mathrm{f}$, making these planets "water worlds", even if many (Earth) oceans of $\mathrm{H}_{2} \mathrm{O}$ were lost (Bolmont et al. 2017). The maximum $\mathrm{H}_{2} \mathrm{O}$ fraction is $\approx 50 \%$ when the planets reach their isolation mass at the $\mathrm{H}_{2} \mathrm{O}$ iceline.

The model that we outlined here can also be applied to compact systems around higher mass stars. Early- to mid-type M stars exhibit a high concentration of planets at close distances (Dressing \& Charbonneau 2015; Muirhead et al. 2015). In addition, Kepler has found many compact systems of superEarth planets around solar-mass stars (Petigura et al. 2013). In our model the typical planet mass scale is $\sim h^{3} M_{\star}$ (Eq. (15)), which would correspond to $\sim 10 M_{\oplus}$ for solar-mass stars (still assuming $h=0.03$ ). The observation that fewer large (Neptunelike) planets are found around $\mathrm{M}$ stars as compared to FGK-stars (Mulders et al. 2015) therefore agrees with one of the pillars of our scenario: that the upper mass limit of rocky planets is set by the pebble isolation mass.

The key characteristic of our model is that close-in planets are formed by tapping the pebble flux that originated at large distances $(\sim 10-100 \mathrm{au})$. This assumes that the disk pressure profile monotonically decreases with radius (no local pressure maxima). Moreover, we have argued that the natural location to rapidly form a planet is the $\mathrm{H}_{2} \mathrm{O}$ iceline, which for solar-type stars is located at $\sim 2-3 \mathrm{au}$. When growth at the iceline proceeds rapidly, a $\sim 10 M_{\oplus}$ core forms a large pre-planetary $\mathrm{H} / \mathrm{He}$-atmosphere, which can collapse into a giant planet upon reaching the critical core mass, and a $\mathrm{H}_{2} \mathrm{O}$-enriched atmosphere would greatly accelerate this process (Venturini et al. 2015). When indeed a giant planet would form rapidly at the snowline, it terminates the pebble flux to the inner disk, starving it from planet-building material and rendering it dry (Morbidelli et al. 2016). Hence, we expect a dichotomy: when giant planet formation fails, pebbles can drift across the iceline to aid the growth of super-Earths and mini-Neptunes. Conversely, when a giant planet forms at the iceline, we expect a dearth of planetary building blocks in the inner disk. Therefore, the close-in super-Earth population found by Kepler and the cold Jupiter populations found chiefly by radial velocity surveys should be anti-correlated ${ }^{5}$. This prediction could be tested with future exoplanet surveys.

Acknowledgements. We thank Allona Vazan, Anders Johansen, Andrius Popovas, Carsten Dominik, Jean-Michel Désert, Samaya Nissanke, and Sebastiaan Krijt for useful discussions and the referee for a critical report that sharpened the presentation. The authors are supported by The Netherlands Organization for Scientific Research (NWO; VIDI project 639.042.422).

\section{References}

Banzatti, A., Pinilla, P., Ricci, L., et al. 2015, ApJ, 815, L15 Batygin, K., \& Morbidelli, A. 2013, AJ, 145, 1

Benítez-Llambay, P., Masset, F., Koenigsberger, G., \& Szulágyi, J. 2015, Nature, 520,63

Birnstiel, T., Andrews, S. M., \& Ercolano, B. 2012, A\&A, 544, A79

Bolmont, E., Selsis, F., Owen, J. E., et al. 2017, MNRAS, 464, 3728

Birnstiel, T., Dullemond, C. P., \& Brauer, F. 2010, A\&A, 513, A79

Bourrier, V., Ehrenreich, D., Wheatley, P. J., et al. 2017, A\&A, 599, L3

Carrera, D., Johansen, A., \& Davies, M. B. 2015, A\&A, 579, A43

Chatterjee, S., \& Tan, J. C. 2014, ApJ, 780, 53

de Wit, J., Wakeford, H. R., Gillon, M., et al. 2016, Nature, 537, 69

Drążkowska, J., Alibert, Y., \& Moore, B. 2016, A\&A, 594, A105

Dressing, C. D., \& Charbonneau, D. 2015, ApJ, 807, 45

Dubrulle, B., Morfill, G., \& Sterzik, M. 1995, Icarus, 114, 237

Frank, J., King, A., \& Raine, D. 1992, Accretion power in astrophysics.

Gillon, M., Jehin, E., Lederer, S. M., et al. 2016, Nature, 533, 221

Gillon, M., Triaud, A. H. M. J., Demory, B.-O., et al. 2017, Nature, 542, 456

Goldreich, P., \& Ward, W. R. 1973, ApJ, 183, 1051

Güttler, C., Blum, J., Zsom, A., Ormel, C. W., \& Dullemond, C. P. 2010, A\&A, 513, A56

Hansen, B. M. S., \& Murray, N. 2012, ApJ, 751, 158

Hartmann, L. 2009, Accretion Processes in Star Formation, second edition (Cambridge University Press)

Hu, X., Zhu, Z., Tan, J. C., \& Chatterjee, S. 2016, ApJ, 816, 19

Ida, S., \& Guillot, T. 2016, A\&A, 596, L3

Ida, S., Guillot, T., \& Morbidelli, A. 2016, A\&A, 591, A72

Izidoro, A., Morbidelli, A., Raymond, S. N., Hersant, F., \& Pierens, A. 2015, A\&A, 582, A99

Johansen, A., Oishi, J. S., Low, M., et al. 2007, Nature, 448, 1022

Johansen, A., Youdin, A., \& Mac Low, M. 2009, ApJ, 704, L75

Kley, W., \& Nelson, R. P. 2012, ARA\&A, 50, 211

Kretke, K. A., \& Levison, H. F. 2014, AJ, 148, 109

Krijt, S., Ormel, C. W., Dominik, C., \& Tielens, A. G. G. M. 2016, A\&A, 586, A20

Lambrechts, M., \& Johansen, A. 2012, A\&A, 544, A32

Lambrechts, M., \& Johansen, A. 2014, A\&A, 572, A107

Lambrechts, M., Johansen, A., \& Morbidelli, A. 2014, A\&A, 572, A35

Lee, M. H., \& Peale, S. J. 2002, ApJ, 567, 596

Levison, H. F., Kretke, K. A., \& Duncan, M. J. 2015, Nature, 524, 322

Lin, D. N. C., \& Papaloizou, J. C. B. 1993, in Protostars and Planets III, eds.

E. H. Levy, \& J. I. Lunine, 749

Lithwick, Y., \& Wu, Y. 2012, ApJ, 756, L11

Liu, B., Ormel, C. W., \& Lin, D. N. C. 2017, A\&A, 601, A15

Lodders, K. 2003, ApJ, 591, 1220

Lopez, E. D., Fortney, J. J., \& Miller, N. 2012, ApJ, 761, 59

Luger, R., Sestovic, M., Kruse, E., et al. 2017, Nat. Astron., 1, 0129

Lynden-Bell, D., \& Pringle, J. E. 1974, MNRAS, 168, 603

Manara, C. F., Testi, L., Natta, A., \& Alcalá, J. M. 2015, A\&A, 579, A66

McNeil, D., Duncan, M., \& Levison, H. F. 2005, AJ, 130, 2884

Morbidelli, A., Lambrechts, M., Jacobson, S., \& Bitsch, B. 2015, Icarus, 258, 418

Morbidelli, A., Bitsch, B., Crida, A., et al. 2016, Icarus, 267, 368

5 Izidoro et al. (2015) arrived at the same prediction from a different context. In their scenario, giant planets block the migration of superEarths and mini-Neptunes, which formed even farther out in the outer disk. 
Muirhead, P. S., Mann, A. W., Vanderburg, A., et al. 2015, ApJ, 801, 18

Mulders, G. D., \& Dominik, C. 2012, A\&A, 539, A9

Mulders, G. D., Pascucci, I., \& Apai, D. 2015, ApJ, 814, 130

Nakagawa, Y., Sekiya, M., \& Hayashi, C. 1986, Icarus, 67, 375

Ogihara, M., \& Kobayashi, H. 2013, ApJ, 775, 34

Ogihara, M., Morbidelli, A., \& Guillot, T. 2015, A\&A, 578, A36

Okuzumi, S., \& Ormel, C. W. 2013, ApJ, 771, 43

Ormel, C. W. 2017, in Formation, Evolution, and Dynamics of Young Solar Systems, eds. M. Pessah, \& O. Gressel, Proc. Sant Cugat Forum on Astrophysics (Springer)

Ormel, C. W., \& Klahr, H. H. 2010, A\&A, 520, A43

Paardekooper, S.-J., Baruteau, C., \& Kley, W. 2011, MNRAS, 410, 293

Paardekooper, S.-J., Rein, H., \& Kley, W. 2013, MNRAS, 434, 3018

Papaloizou, J. C. B. 2015, Int. J. Astrobiol., 14, 291

Pérez, L. M., Chandler, C. J., Isella, A., et al. 2015, ApJ, 813, 41

Petigura, E. A., Marcy, G. W., \& Howard, A. W. 2013, ApJ, 770, 69

Rafikov, R. R., \& De Colle, F. 2006, ApJ, 646, 275

Reiners, A., Basri, G., \& Christensen, U. R. 2009, ApJ, 697, 373

Ricci, L., Testi, L., Natta, A., Scholz, A., \& de Gregorio-Monsalvo, I. 2012, ApJ, 761, L20
Ros, K., \& Johansen, A. 2013, A\&A, 552, A137

Saito, E., \& Sirono, S.-I. 2011, ApJ, 728, 20

Sato, T., Okuzumi, S., \& Ida, S. 2016, A\&A, 589, A15

Schäfer, U., Yang, C.-C., \& Johansen, A. 2017, A\&A, 597, A69

Schoonenberg, D., \& Ormel, C. W. 2017, A\&A, 602, A21

Simon, J. B., Armitage, P. J., Li, R., \& Youdin, A. N. 2016, ApJ, 822, 55 Stelzer, B., Marino, A., Micela, G., López-Santiago, J., \& Liefke, C. 2013, MNRAS, 431, 2063

Stevenson, D. J., \& Lunine, J. I. 1988, Icarus, 75, 146

Tamayo, D., Rein, H., Petrovich, C., \& Murray, N. 2017, ApJ, 840, L19

Tanaka, H., Takeuchi, T., \& Ward, W. R. 2002, ApJ, 565, 1257

Terquem, C., \& Papaloizou, J. C. B. 2007, ApJ, 654, 1110

Testi, L., Birnstiel, T., Ricci, L., et al. 2014, Protostars and Planets VI, 339

Venturini, J., Alibert, Y., Benz, W., \& Ikoma, M. 2015, A\&A, 576, A114

Weidenschilling, S. J. 1977, MNRAS, 180, 57

Yang, C.-C., Johansen, A., \& Carrera, D. 2017, A\&A, in press DOI: $10.1051 / 0004-6361 / 201630106$

Youdin, A. N., \& Goodman, J. 2005, ApJ, 620, 459

Zhu, Z., Nelson, R. P., Dong, R., Espaillat, C., \& Hartmann, L. 2012, ApJ, 755, 